\title{
CYP709B3, a cytochrome P450 monooxygenase gene involved in salt tolerance in Arabidopsis thaliana
}

\author{
Guohong Mao ${ }^{1,2}$, Timothy Seebeck ${ }^{1,2}$, Denyse Schrenker ${ }^{1,3}$ and Oliver $Y u^{1,2^{*}}$
}

\begin{abstract}
Background: Within the Arabidopsis genome, there are 272 cytochrome P450 monooxygenase (P450) genes. However, the biological functions of the majority of these P450s remain unknown. The CYP709B family of P450s includes three gene members, CYP709B1, CYP709B2 and CYP709B3, which have high amino acid sequence similarity and lack reports elucidating biological functions.

Results: We identified T-DNA insertion-based null mutants of the CYP709B subfamily of genes. No obvious morphological phenotypes were exhibited under normal growth conditions. When the responses to ABA and salt stress were studied in these mutants, only the cyp70963 mutant showed sensitivity to ABA and salt during germination. Under moderate salt treatment (150 mM NaCl), cyp70963 showed a higher percentage of damaged seedlings, indicating a lower tolerance to salt stress. CYP709B3 was highly expressed in all analyzed tissues and especially high in seedlings and leaves. In contrast, CYP709B1 and CYP709B2 were highly expressed in siliques, but were at very low levels in other tissues. Under salt stress condition, CYP709B3 gene expression was induced after $24 \mathrm{hr}$ and remained at high expression level. Expression of the wild type CYP709B3 gene in the cyp709b3 mutant fully complemented the salt intolerant phenotype. Furthermore, metabolite profiling analysis revealed some differences between wild type and cyp709b3 mutant plants, supporting the salt intolerance phenotype of the cyp709b3 mutant.
\end{abstract}

Conclusions: These results suggest that CYP709B3 plays a role in ABA and salt stress response and provides evidence to support the functions of cytochrome P450 enzymes in plant stress response.

Keywords: CYP709B, Salt tolerance, Germination, Expression pattern, Metabolic profile

\section{Background}

Cytochrome P450 monooxygenases (P450s) are universal enzymes present in most organisms from bacteria to plants and humans. They catalyze the oxidation of various substrates through activation of molecular oxygen. All P450s share a common catalytic center; a heme with an iron coordinated to the thiolate of a conserved cysteine. In plants, a large number of P450 genes form a superfamily and play important roles in plant metabolic processes [1-3].

\footnotetext{
* Correspondence: oliver.yu@conagen-inc.com

'Donald Danforth Plant Science Center, 975 North Warson Road, St. Louis, MO 63132, USA

${ }^{2}$ Present address: Conagen Inc., 1005 North Warson Road, St., Louis, MO 63132, USA

Full list of author information is available at the end of the article
}

Plant P450s are generally classified into two main clades: the A-type and the non-A-type. The A-type clade is specific to plants while the non-A-type clade is a divergent group with members showing more similarity to non-plant P450s [4,5]. Plant P450s catalyze biosynthetic steps for a wide range of plant metabolites, including pigments, defense-related compounds, UV protectants, lignin, fatty acids and phytohormones [2,3,6-8].

Plant genomes encode much higher numbers of P450 genes than those of other organisms, reflecting the broad biological functions of P450s in primary and secondary metabolism. In the Arabidopsis thaliana genome, the P450 superfamily contains 272 genes (including 26 pseudogenes), of which 153 are A-type P450s and 93 are non-A-type P450s [3]. Thus far a small number of the 246 putative coding sequences have been associated with

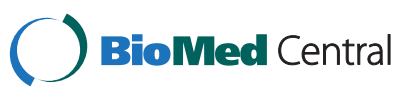


a specific biochemical function [3,7], meaning that the biological functions of the majority of the P450 genes in Arabidopsis remain unknown.

As new technical approaches are developed, the biological functions of more plant P450s have been identified. Expression and co-expression analysis provides clues for functional annotation of P450s in Arabidopsis. For example, Narusaka et al. studied the crosstalk between abiotic and biotic stress responses using a cDNA microarray containing the genes in the cytochrome P450 superfamily [9]. The Werck-Reichhart group generated an extensive co-expression analysis tool for the cytochrome P450 superfamily $[10,11]$. These expression analyses provided novel clues to the functions, metabolic pathways and regulatory networks of individual P450s. Also, based on co-expression analysis, a novel phenolic pathway in pollen development was identified [12]. An abundance of P450 expression data was collected that provides information into the biological functions of P450s in plant development and the responses to chemical and environmental stresses. For example, the P450s CYP71A19, CYP71B19, CYP71B20, CYP71B26, CYP71B28, CYP76C2, CYP86B1, CYP89A9 and CYP94B3 are induced in response to ABA treatments ( 3 to $24 \mathrm{~h}$ ), IAA treatment $(3 \mathrm{~h})$ and osmotic stress $(3 \mathrm{~h})$; patterns similar to those of CYP707A1, which is known to mediate ABA catabolism $[7,13]$. These data are starting points to identify the functions of these P450s in stress response.

Heterologous or in vitro expression has proven a useful tool in unveiling the biochemical functions of P450s. For instance, CYP735A1 and CYP735A2 were identified as cytokinin hydroxylases that catalyze the biosynthesis of trans-Zeatin by using an adenosine phosphateisopentenyltransferase (AtIPT4)/P450 co-expression system in yeast [14]. In addition, the CYP707A1-CYP707A4 genes were functionally expressed in yeast and found to have an ABA 8'-hydroxylase activity [15]. Despite these successes, the membrane-bound nature of $\mathrm{P} 450$ proteins and the presence of $\mathrm{P} 450$ reductases create special challenges for using heterologous systems, and thus have limited the number of P450s with defined enzymatic activity.

Some stress related plant $\mathrm{P} 450$ genes were identified by genetic screening $[7,8]$. For example, cyp $707 a \mathrm{mu}-$ tants exhibited hyperdormancy in seeds and accumulated greater ABA content than wild type, indicating that CYP707 genes regulate ABA catabolism [15-18].

At present, there is limited information about the CYP709B subfamily. Expression data showed that some of the $C Y P 709 B$ genes were regulated by phytohormones [19] and circadian rhythm [20]. No enzymatic activity was identified by using the yeast expression system $[14,21,22]$. In this report, using genetic screening, we identified the null mutants of the CYP709B genes and compared the phenotypes in germination and salt tolerance. Only the cyp $709 b 3$ mutant exhibited the ABA and salt sensitive phenotypes. Expression of the wild type CYP709B3 gene in the cyp709b3 mutant fully complemented the salt intolerance phenotype. The possible function of CYP709B3 in salt tolerance is also discussed.

\section{Results}

Identification of T-DNA insertion mutants of CYP709B

\section{family genes}

The CYP709B subfamily belongs to non-A-type cytochrome P450s and includes three gene members: $C Y P$ 709B1, CYP709B2 and CYP709B3. The putative proteins share high identity at the amino acid level (Additional file 1). The CYP709B1 (At2g46960) and CYP709B2 (At2g 46950) genes are located on chromosome 2 and both have 5 exons and 4 introns. Physically, they are $759 \mathrm{bp}$ apart in genomic sequence. CYP709B3 (At4g27710) is located on chromosome 4 and also has 5 exons and 4 introns.

We identified T-DNA insertion mutants in each of the CYP709B subfamily members, all in Columbia-0 (Col-0) background. SALK_021290C (cyp709b1) has an insertion in the promoter of the CYP709B1 gene, and SALK_011121 (cyp709b3) has an insertion in the fifth exon of the CYP709B3 gene. We identified two mutant alleles of the CYP709B2 gene, one of which has an insertion in the second exon (SALK_020401, cyp709b2-1) and the other in the third intron (SALK_087806, cyp709b2-2) (Figure 1A). Reverse transcription RT-PCR analysis of the total RNA from mutant leaves or flowers was performed using gene-specific primers. As shown in Figure $1 \mathrm{~B}$, the gene transcripts were not detected in the mutants, demonstrating that all mutants were transcript null.

\section{Tissue-specific expression pattern of CYP709B subfamily genes}

The transcript levels of the $C Y P 709 B$ genes from seedlings, inflorescences, rosette leaves, flowers and siliques were analyzed by a quantitative real time PCR method. CYP709B1 and CYP709B2 showed very low expression levels in seedlings, inflorescences, and rosettes, but had high expression levels in siliques (Figure 2A and B). Transcripts of CYP709B3 were detected in all tested organs and were more abundant in rosette leaves and siliques (Figure 2C). Recently, a batch of microarray data also showed similar expression patterns (http://bbc.botany.utoronto.ca) [23]. As confirmed by our data and by available database information, the $C Y P 709 B 3$ gene is universally expressed while the CYP709B1 and CYP709B2 are highly expressed in mature siliques, indicating that 


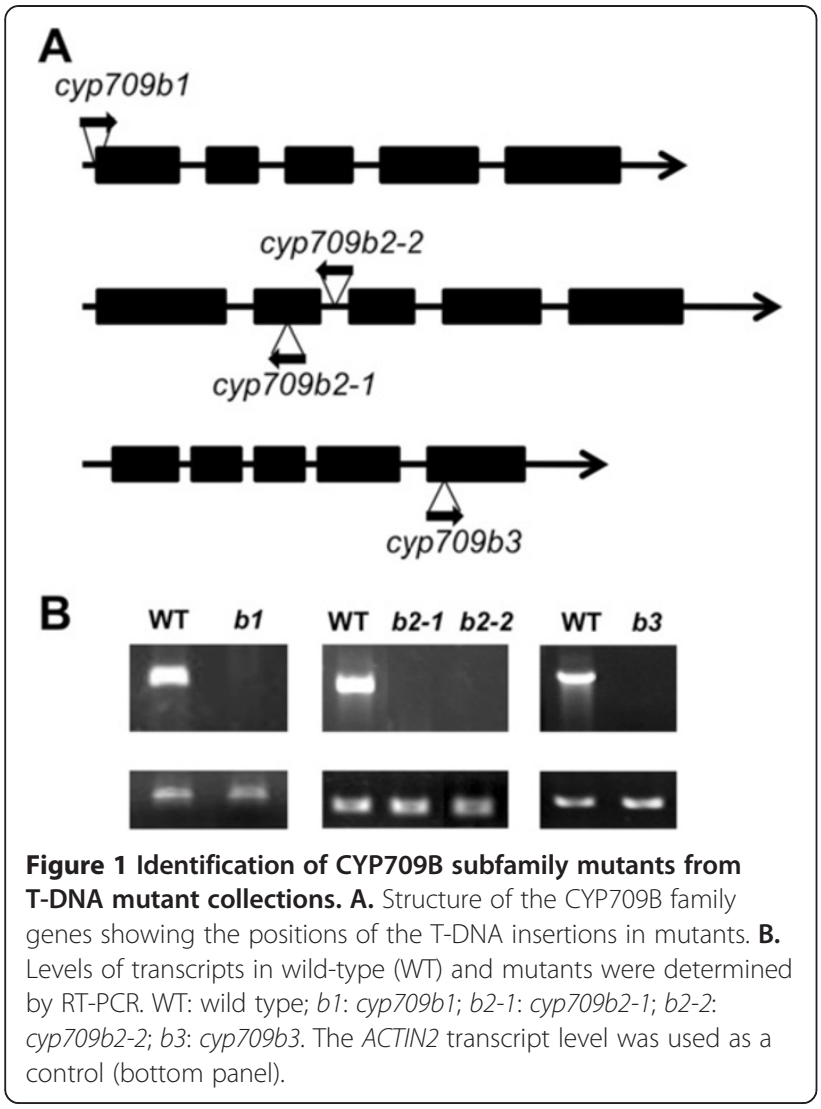

these closely related genes may have different biological functions.

\section{cyp709b3 mutant is sensitive to ABA and salt stress in germination}

The CYP709B T-DNA insertion mutants displayed no visible phenotypic alterations under normal growth conditions. The mature plant height, rosette size, flower, silique and seed were all similar to wild type, suggesting that the CYP709B genes are not essential to vegetative or reproductive growth and development. Since all three genes were highly expressed in siliques and seeds, we speculated that loss-of-function of the CYP709B genes would lead to seed-related phenotypes, such as in seed dormancy and/or germination. To assess whether any of the CYP709B genes play roles in controlling seed germination, we performed germination assays using wild type and cyp709b mutants. To determine whether the mutation affects seed germination in response to ABA, wild type and mutant seed was sown on filter paper saturated with water and different concentrations of ABA. After stratification at $4^{\circ} \mathrm{C}$ for 2 days, germination was scored daily. Without ABA treatment, all seeds germinated; reaching around $100 \%$ at day 2 . Germination of the cyp709b3 mutant was inhibited by application of ABA (Figure 3A). In contrast, seed of the other two

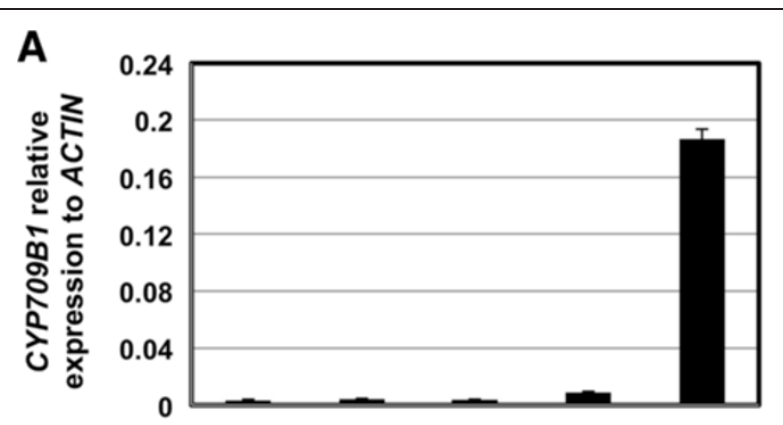

B
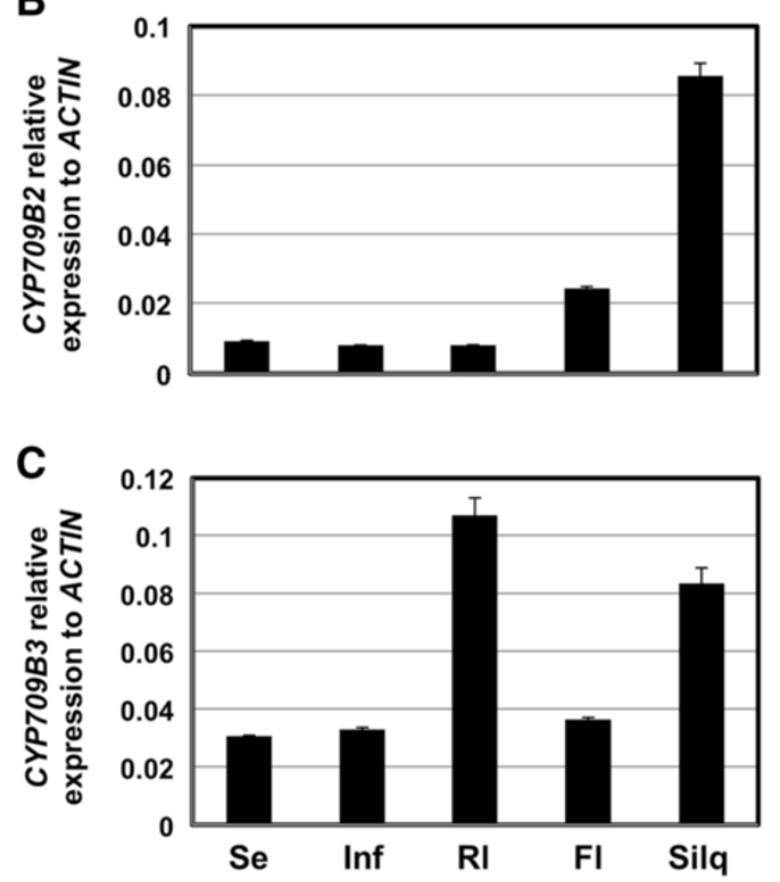

Figure 2 Tissue-specific expression in wild type Arabidopsis. A: CYP709B1. B: CYP709B2. (C) CYP709B3. Se: 12-day-old seedlings; Inf: inflorescences; Rl: rosette leaves; FL: opening flowers; Silq: siliques (4-10 DAP). Real time PCR was performed to detect the level of transcripts in various organs of wild type. ACTIN2 was used as internal control. Values are the means \pm SE of three replicates.

mutants germinated similarly to wild type under ABA treatment. In the presence of $1.5 \mu \mathrm{M} A B A$, the germination of cyp $709 b 3$ was dramatically delayed. At day 5, around $90 \%$ of the wild type seeds had germinated, while only $55 \%$ of the cyp $709 b 3$ seeds had. The delayed germination in cyp709b3 seeds was presumably due to increased sensitivity to exogenous ABA.

cyp709b3 seed germination was also more sensitive to salt stress than seeds of wild type and the other mutants. In the presence of 150 or $200 \mathrm{mM} \mathrm{NaCl}$, the germination of cyp $709 b 3$ seeds was delayed. At day 3, only $83 \%$ and $43 \%$ of the cyp $709 b 3$ seeds germinated in the presence of 150 and $200 \mathrm{mM} \mathrm{NaCl}$, respectively. In contrast, $98 \%$ and $70 \%$ of the wild type seeds had germinated. 


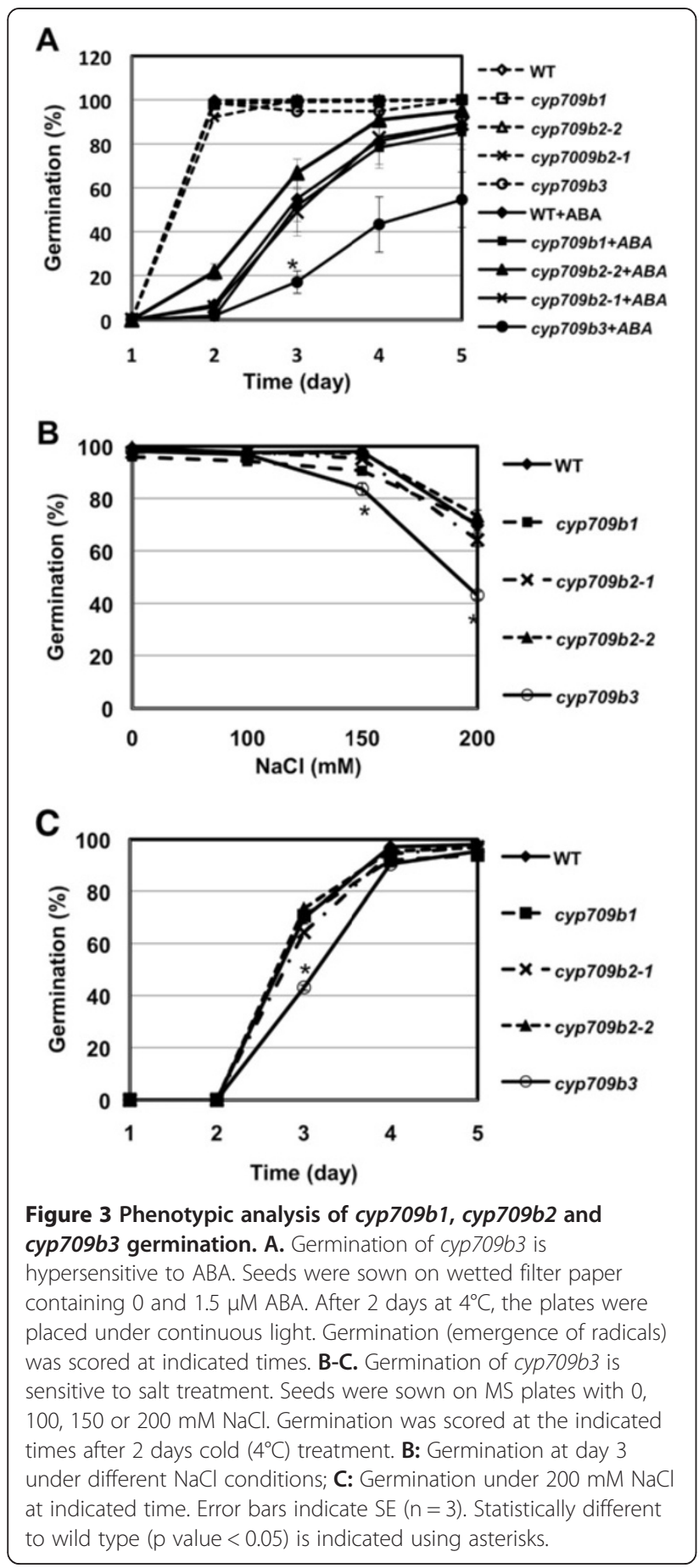

The cyp709b2-1, cyp709b2-2 and cyp709b1 mutant seeds exhibited similar germination rates as wild type (Figure 3 $\mathrm{B}$ and C). Although the germination of cyp $709 \mathrm{b3}$ seeds was inhibited at day 3 under $200 \mathrm{mM} \mathrm{NaCl}$ treatment, it was similar to the wild type germination rate at day 4 . These results indicate that only the cyp $709 b 3$ mutant is sensitive to ABA and salt during germination. cyp709b3 mutant shows a salt intolerance phenotype

Furthermore, we detected the plant growth phenotypes under stress conditions. Root growth under salt and mannitol treatment was analyzed, and no significant differences between the mutants and wild type were revealed. However, when cyp $709 b 3$ mutant seedlings were kept on salt plates, more seedlings became bleached and dead. To evaluate the salt tolerance of the cyp $709 b 3 \mathrm{mu}-$ tant, the seeds were germinated on MS agar plates for 4 days at which time the seedlings were transferred onto MS agar plates supplemented with $100 \mathrm{mM}, 150 \mathrm{mM}$ or $200 \mathrm{mM} \mathrm{NaCl}$. The rate of dead seedlings was scored daily (Figure 4A). When exposed to $150 \mathrm{mM} \mathrm{NaCl}$, more cyp $709 b 3$ seedlings were damaged than wild type (Figure 4B) and other mutants (Figure 4C). From day $5-7$, around $70-77 \%$ of the cyp709b3 seedlings were dead under $150 \mathrm{mM} \mathrm{NaCl}$ treatment, compared to 26$30 \%$ of wild type seedlings (Figure 4A). cyp $709 b 1$ and cyp709b2 mutants exhibited similar rates of dead seedlings as wild type (Figure 4C). Exposure to $100 \mathrm{mM}$ $\mathrm{NaCl}$ caused fewer seedlings to be damaged in both wild type and cyp709b3 mutants. Under $200 \mathrm{mM} \mathrm{NaCl}$ treatment, almost all wild type and cyp $709 b 3$ seedlings were died at day 4. In other words, there was no significant difference between wild type and mutants under mild $(100 \mathrm{mM} \mathrm{NaCl})$ or severe salt $(200 \mathrm{mM} \mathrm{NaCl})$ treatment.

To further confirm the salt stress phenotype, 12-dayold plants grown in soil were irrigated with $150 \mathrm{mM}$ $\mathrm{NaCl}$. After 2 weeks of treatment, cyp709b3 mutant plants started showing serious damage compared to wild type and other mutants. As shown in Figure 4D, all plants presented yellowed leaves after 3 weeks of salt treatment; however, cyp 70963 plants presented more dead plants (fully bleached) under the same growth conditions. There were no differences between any of the genotypes under normal conditions. These results indicate that cyp $709 b 3$ mutant seedlings and plants are more sensitive to moderate salt stress $(150 \mathrm{mM} \mathrm{NaCl})$.

\section{Expression of wild type CYP709B3 gene can rescue the salt sensitive phenotype}

A complementation experiment was performed to further verify the function of CYP709B3. First, the promoter region was obtained by amplifying the 1547-bp region upstream of the ATG start codon. A Pro ${ }_{C Y P 709 B 3}$ : GUS fusion construct was generated and transformed into wild-type plants. GUS activity was detected in whole seedlings, rosette leaves, siliques and flowers (Figure 5A-D). GUS reporter staining revealed a highly similar expression pattern with this promoter fragment as real-time PCR results did for CYP709B3 (Figure 2C). Furthermore, a native CYP709B3 promoter construct

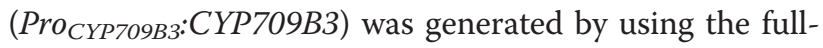
length $C Y P 709 B 3$ genomic DNA that included the same 


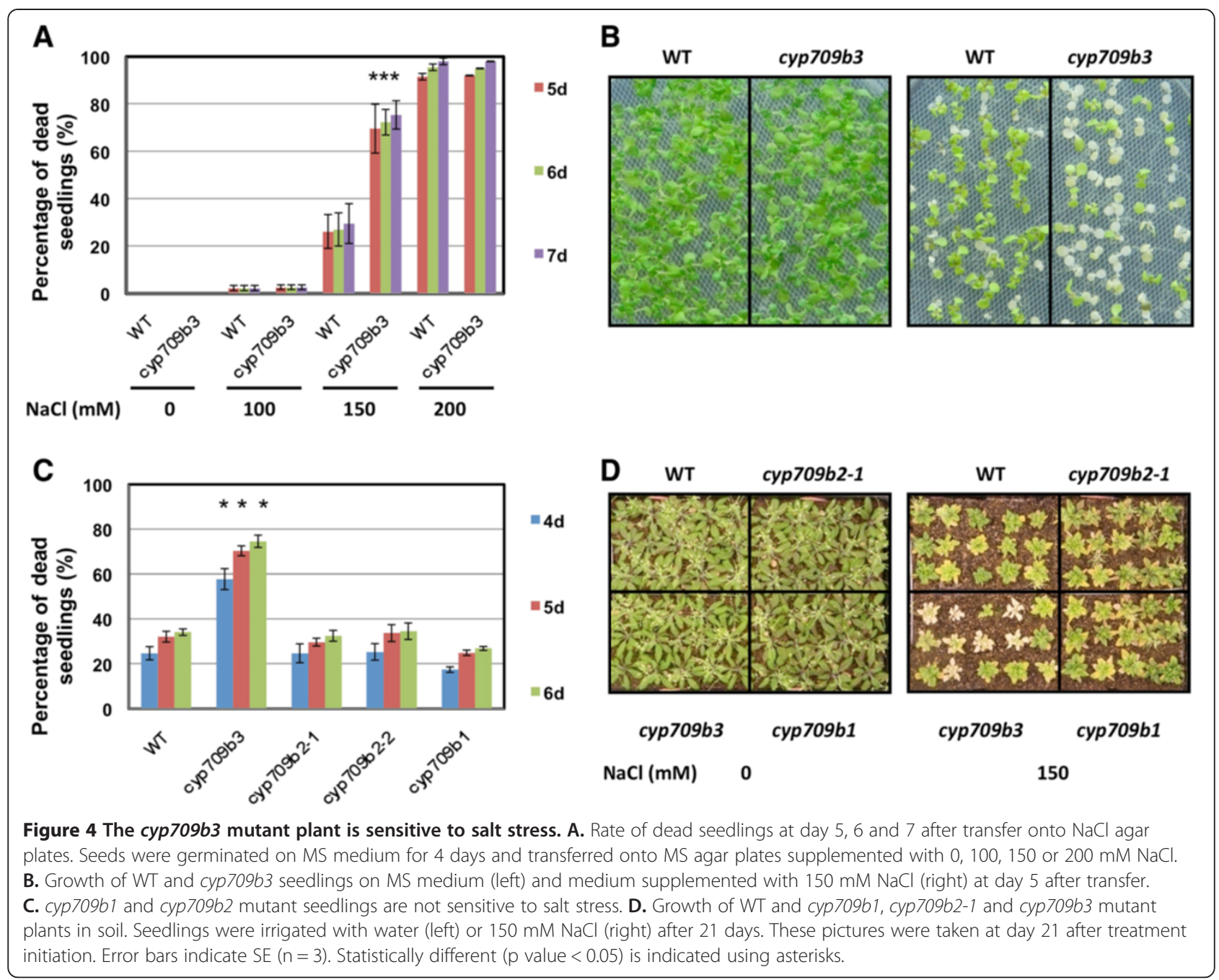

promoter region. When the construct was transformed into the cyp709b3 T-DNA insertion plants, the wild type gene fully rescued the salt sensitive phenotype displayed in the cyp709b3 plants (Figure 5E). Three independent homozygous transgenic lines (11-4, 18-8 and 40-2) were selected for detailed analyses. As shown in Figure 5E, these three transgenic lines had similar rates of bleached seedlings as wild type when challenged with150 mM NaCl treatment. The CYP709B3 gene expression level was similar between the transgenic lines and wild type (Figure 5F). We also found wild type CYP709B3 gene can recue ABA sensitive germination phenotype in transgenic line (Additional file 2). These results strongly support the function of CYP709B3 in salt tolerance.

\section{Gene expression under salt stress}

The requirement of CYP709B3 in ABA and salt sensitive responses prompted us to investigate whether CYP709B3 was required for stress-regulated gene expression. When
CYP709B3 gene expression under $150 \mathrm{mM} \mathrm{NaCl}$ stress was checked, we found that CYP709B3 gene expression was not induced at the early stage of salt treatment; however, the expression was induced after $24 \mathrm{~h}$ and remained high at later time points (Figure 6A). Although CYP709B2 gene expression was dramatically lower than CYP709B3 in seedlings, CYP709B2 gene expression was also induced by salt stress, peaking after $3 \mathrm{hr}$ of $150 \mathrm{mM} \mathrm{NaCl}$ treatment and then dropping down to basal level (Figure 6A). CYP709B1 expresses at very low levels in seedlings and was not detected under salt treatment.

Furthermore, we found that stress-regulated genes (KIN2, RD29A and RD29B) in the cyp709b3 mutant were not significantly altered compared to wild type. $D R E B 1 A$ and ERD10 expression was slightly decreased at 1 hour after $150 \mathrm{mM} \mathrm{NaCl}$ treatment (Figure 6B-F), indicating that the cyp709b3 mutant did not substantially impair the up-regulation of stress-regulated genes. 

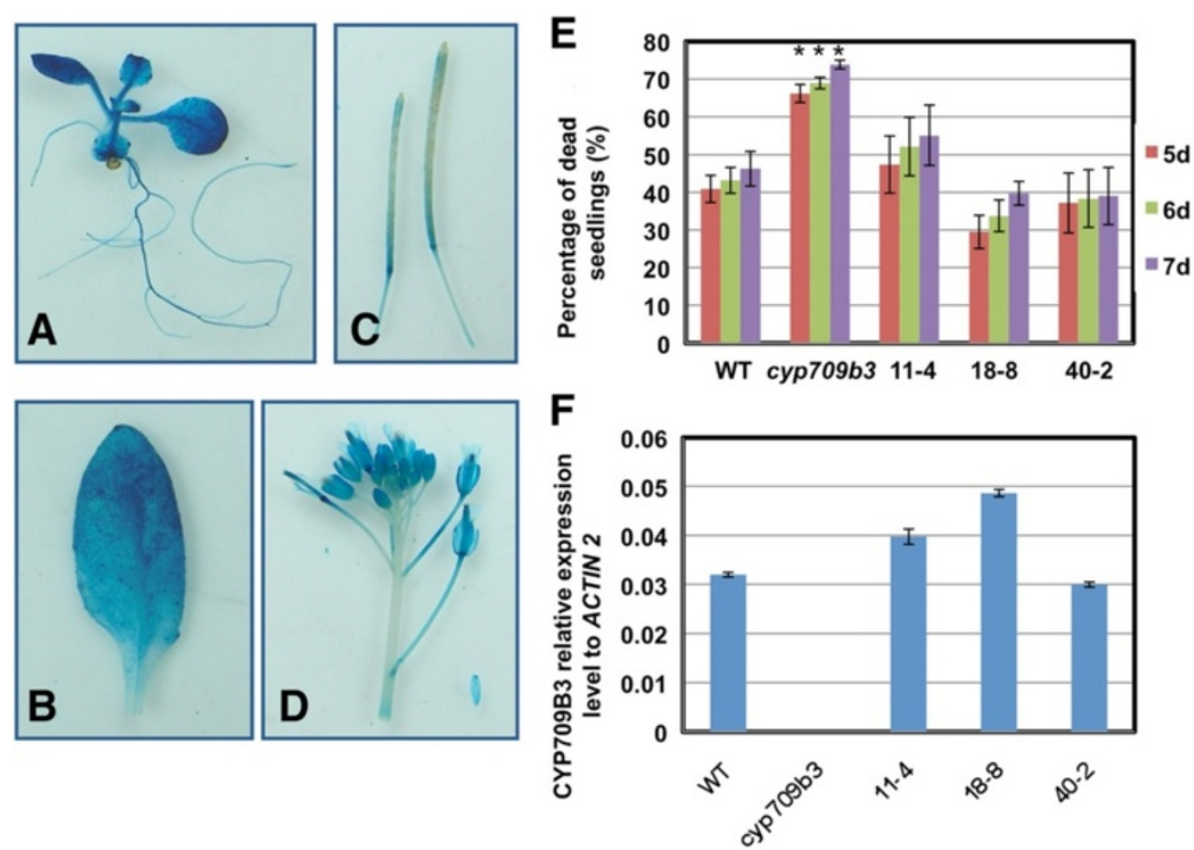

Figure 5 Expression of wild type CYP709B3 gene in cyp709b3 mutant can rescue salt intolerance phenotype. A-D. Expression pattern of

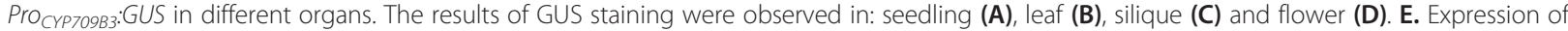
a wild type CYP709B3 gene can rescue the salt intolerance phenotype. Wild type and three independent Pro CYP709B3:CYP709B3 transgenic homozygous lines (11-4, 18-8 and 40-2) in cyp709b3 mutant background were treated by $150 \mathrm{mM} \mathrm{NaCl}$. The seedlings were counted at the indicated times. F. Analysis of the expression level of CYP709B3 in seedlings from wild type, cyp709b3 and independent transgenic homozygous lines (11-4, 18-8 and 40-2). ACTIN2 was used as internal control. Values are the means \pm SE of three replicates. Statistically different ( $p$ value $<$ $0.05)$ is indicated using asterisks.

\section{ABA content is not affected in the cyp709b3 mutant}

The phytohormone ABA is key in regulating plant stress responses and plays important roles in seed germination [24]. Since the cyp709b3 mutant seed is sensitive to both $\mathrm{ABA}$ and salt in germination, we analyzed the endogenous ABA content during seed imbibition and salt stress. During seed imbibition, the change in the endogenous ABA level in the cyp709b3 mutant is the same as in wild type seeds (Figure 7A). Endogenous ABA content decreased to the same levels 12 and 24 hours after imbibition in both cyp709b3 mutant and wild type. After $150 \mathrm{mM} \mathrm{NaCl}$ treatment, the ABA content reached a similar maximal level in both wild type and cyp709b3 seedlings at 6 hours, then dropped down to the basal level 2 day after treatment (Figure 7B). These data clearly demonstrate that CYP709B subfamily genes are not involved in ABA metabolism.

\section{Metabolite profiling under salt stress}

Metabolite profiles of wild type and the cyp709b3 mutant under both normal and salt stress growth condition were determined using global metabolomic analysis (METABOLON Inc.).

All wild type and cyp709b3 seedlings were green for the first 3 days after transferring onto $150 \mathrm{mM} \mathrm{NaCl}$ plates. From day 4 onwards, the cyp709b3 mutant developed more bleached and dying seedlings than wild type (Figure 4). Both wild type and cyp709b3 seedlings grew normally on MS plates without salt. Therefore, we collected seedling samples at day 2 (2D) and day 4 (4D) from non-salt treated $(\mathrm{N})$ and salt treated $(\mathrm{S})$ plates. Some seedlings were already dead at day 4; we did not pick up these dead seedlings for metabolite analysis. All extracted samples were analyzed by LC/MS and GC/MS. The identified 163 metabolites contain amino acids, carbohydrates, lipids, cofactors, prosthetic groups, electron carriers, nucleotides, peptides, hormones and secondary metabolites.

The metabolomes of both wild type and cyp709b3 were strongly affected by the salt treatment; however, the differences between the lines at similar treatments were relatively subtle. Of the 163 compounds tested, there were no obvious indications of a substrate or product whose presence was associated absolutely with the presence of the mutation (Additional file 3). Nonetheless, some trends did suggest biologically relevant genotype-related differences, both in the absence and presence of salt stress. Of the 163 compounds tested, $61-75 \%$ differed significantly $(p<0.05)$ in the non-salt (N) vs. salt (S) tests. This is in sharp contrast to the 

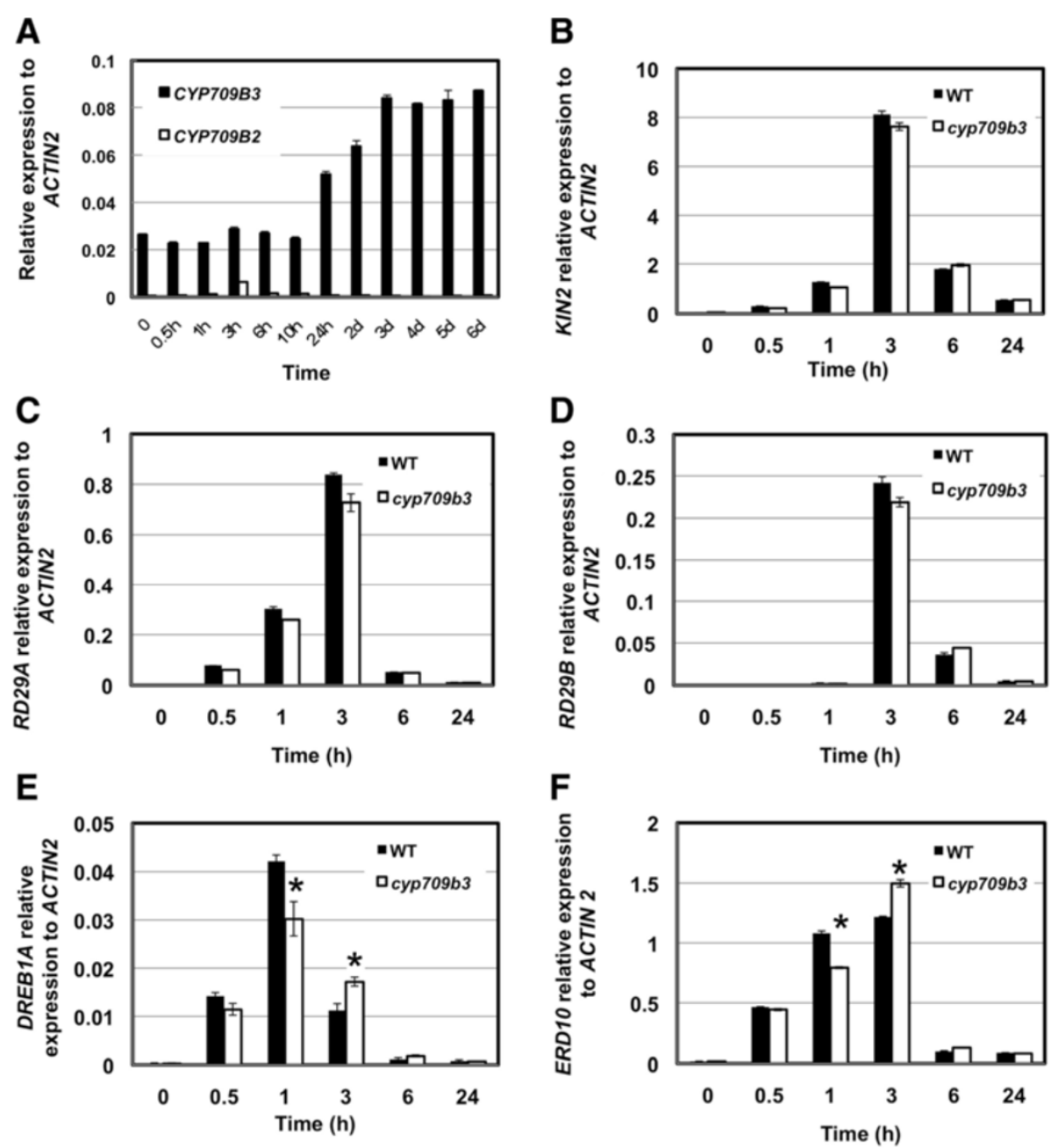

Figure 6 Gene expression analysis in wild type and mutants under $150 \mathrm{mM} \mathrm{NaCl}$ treatment. Seeds were germinated on MS medium for 4 days and transferred to MS medium supplemented with $150 \mathrm{mM} \mathrm{NaCl}$. Seedlings were harvested at indicated times. Gene expression was detected by quantitative real time PCR. A. CYP709B3 and CYP709B2 gene expression in wild type seedlings under $150 \mathrm{mM} \mathrm{NaCl}$ treatment. Expression of stress response genes: KIN2 (B), RD29A (C), RD29B (D), DREB1A (E), ERD10 (F) in seedlings from $150 \mathrm{mM} \mathrm{NaCl-treated} \mathrm{wild} \mathrm{type} \mathrm{and}$ cyp709b3 mutant. ACTIN2 was used as internal control. Error bars indicate SE $(n=3)$. Statistically different $(p$ value $<0.05)$ is indicated using asterisks.

time-related differences within either line (2D vs. 4D), in which $23-33 \%$ of the compounds were different and especially to the genotype-related differences from $6-25 \%$ within treatments (MUT vs. WT) (Additional file 4).

The very large number of compounds altered by salt stress illustrates the profound effect of salt stress on Arabidopsis seedlings. Many of these changes were greater than 5 -fold in magnitude, and they included compounds expected to be induced during salt stress (proline and histidine, among many amino acids, glutathione, and GABA).

More general metabolomic perturbations were observed in the non-stressed mutant plants. These include several indications of oxidative and ammonia stress. For example, $\mathrm{NAD}^{+}$and dehydroascorbate, two compounds in pathways which supply intermediates for the remediation of oxidative stress, were lower in non-stressed mutant plants, while ophthalmate, gamma-glutamylglutamate, and beta-alanine were higher.

The large number of strong changes of metabolites tends to overpower any mutant effects. Almost all affected compounds behaved in a similar manner between WT and mutant, in both direction and magnitude of change. However, a few exceptions can be noted. Mutant plants at Day 4 were not able to maintain the induction of spermidine and pantothenate, which could lead to a shortage of $\mathrm{CoA}$, an important cofactor in a wide range of reactions.

Significant changes in several compounds indicate increased cellular damage in the cyp709b3 plants. While 

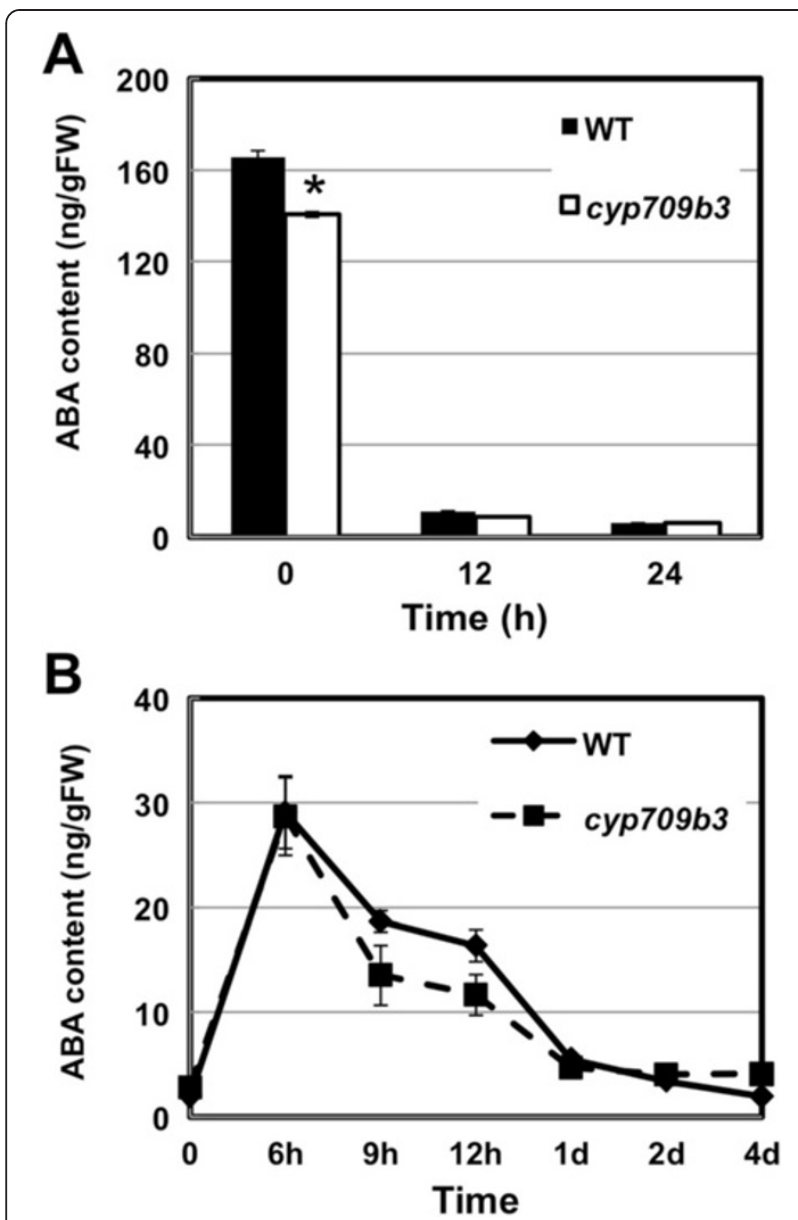

Figure 7 The endogenous level of $A B A$ in wild type and mutants. A. The change of endogenous ABA levels in wild type and cyp709b3 mutant during seed imbibition. The seeds were collected at 0,12 and $24 \mathrm{~h}$ after imbibition. B. Endogenous ABA level in seedlings from $150 \mathrm{mM} \mathrm{NaCl}$-treated seedlings from wild type and cyp709b3 at indicated times. Values are the means \pm SE of three replicates. Statistically different ( $p$ value $<0.05$ ) is indicated using asterisks.

these compounds respond to salt stress in a similar direction in both WT and mutant, the magnitude of the response is significantly stronger in the mutant. For example, the lysolipids are markers of lipolysis in membranes and of membrane damage. All three 1-palmitoyllysolipids showed a similar pattern of stronger induction in cyp 709b3 4D salt-treated plants (Figure 8A, B and C). Likewise, 1-methyladenosine and pseudouridine are markers of nucleic acid turnover; both these compounds are modified post-transcriptionally, and thus represent macromolecular degradation. As shown in Figure 8D and E, both accumulated in the cyp709b3 mutant at 4D after salt treatment. N6-acetyllysine, a product of protein breakdown, also increased in treated cyp $709 \mathrm{b3}$ 4D samples (Figure 8F). Additionally, all the aromatic amino acids exhibited similar patterns of increased salt- response, which may reflect protein turnover (Figure 8G, $\mathrm{H}$ and I). These results indicate that some changes of metabolism under salt stress are related to the cyp 70963 salt intolerance phenotype.

\section{Discussion}

In Arabidopsis, a minority of the P450s have been characterized in detail, with the biochemical function of only a few being fully elucidated $[7,10]$. More than 200 genes remain uncharacterized. Several approaches are being used to study these unknown genes, such as expression and co-expression analysis, genetic screening, and heterologous expression assays.

\section{CYP709B3 plays roles in salt tolerance}

Gene expression patterns identify genes with correlated functions during plant development, or in response to various stimuli. Recently, gene expression profiling and co-expression analysis of the cytochrome P450 superfamily in Arabidopsis was analyzed using cDNA microarrays $[9,11]$. The expression profiling of the CYP709B subfamily was reported in several publications. For example, Duan et al. compared the gene expression profiles of flower and leaf tissues of both $\mathrm{Col}$ and Ler Arabidopsis ecotypes using P450 microarrays [25]. They found that CYP709B2 gene expression in flowers is higher in the Ler ecotype than in the Col ecotype. Furthermore, some of the gene members in the CYP709B subfamily can be regulated by phytohormones: Auxin up-regulates $C Y P 709 B 2$ expression and brassinosteriod down-regulates CYP709B3 expression at later times after treatment [19]. In another study, expression of the CYP709B3 gene showed circadian regulation [20].

In this report, we detected the expression patterns of the three members of the CYP709B subfamily. The results revealed different expression patterns of the genes in the various organs examined. CYP709B3 was expressed universally, but was expressed at the highest levels in leaves and siliques. CYP709B1 and CYP709B2 were highly expressed in siliques but weakly expressed in other examined organs (Figure 2). Furthermore, we found that CYP709B3 expression can be induced by salt stress and continually induced after $24 \mathrm{~h}$ of treatment. While expression of CYP709B2 in seedlings is very low, it was also induced by salt stress. In these experiments, CYP709B1 expression was not detected in either the salt-treated or untreated tissues. These expression profiles indicate that the three $C Y P 709 B$ genes may have divergent functions in plant development or stress response.

Several research groups have tried to identify the enzymatic functions of CYP709B subfamily members using heterologous expression systems. Using an adenosine phosphate-isopentenyltransferase (AtIPT4)/P450 


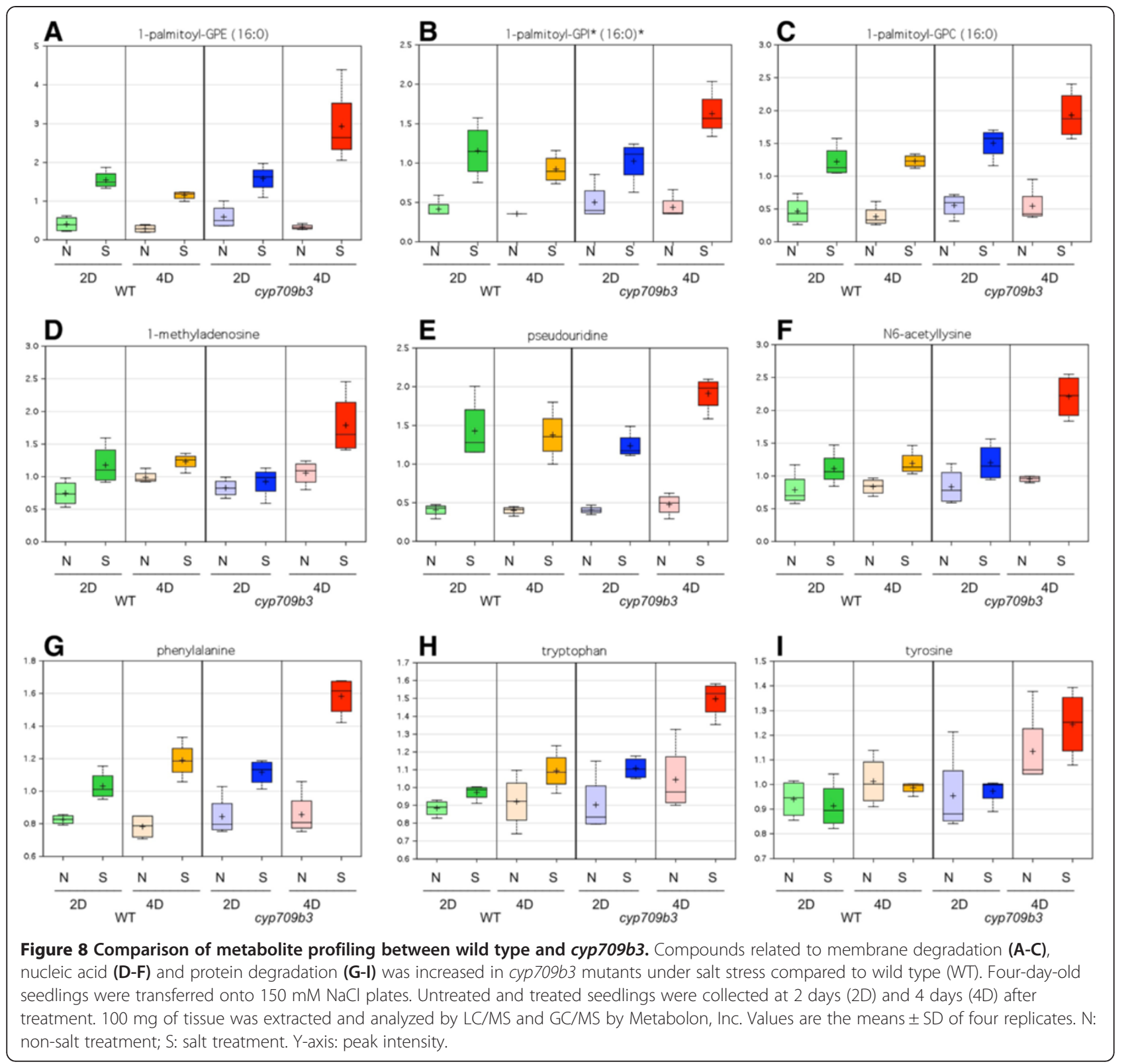

co-expression system in yeast, the CYP735A (formerly named CYP709A) subfamily was identified as a cytokinin hydroxylase that catalyzes the biosynthesis of trans-Zeatin. Phylogenetic analysis of Arabidopsis P450 genes shows that the CYP709B subfamily is a sister group to the CYP735A subfamily. However, no hydroxylase activity was detected for the CYP709Bs in the yeast system [14]. Kandel et al. characterized CYP709C1 from wheat (homolog of the CYP709Bs) as an in-chain hydroxylase [21,22]. Although they tried to detect the same enzymatic activity in the CYP709B subfamily, expression of CYP709B1, CYP709B2, or CYP709B3 in yeast failed to demonstrate any in-chain hydroxylase activity.
To further investigate the functions of the CYP709B subfamily members, we identified T-DNA insertional null mutants. None of the null mutants had a visible morphological alteration, indicating that the CYP709B genes were not essential to plant development. Only the cyp 70963 mutant showed ABA and salt sensitivity in germination (Figure 3) and deficiency in salt tolerance (Figure 4), indicating that CYP709B3 has a unique function in ABA and salt stress responses that is not shared by CYP709B1 and CYP709B2.

CYP709B3 is not directly involved in ABA metabolism or up-regulation of stress-regulated genes in plants

Abscisic acid (ABA) plays pivotal roles in many cellular processes, including seed development, dormancy, 
germination, vegetative growth and environmental stress response. Environmental stresses can dramatically increase ABA levels. The increased ABA levels under stress are due to both active ABA biosynthesis and suppressed ABA degradation. Recently, several published papers have shown that the CYP707A subfamily is involved in ABA metabolism [15]. In the Arabidopsis genome, there are four CYP707A genes; CYP707A1-4. Expression of CYP707A2 is specifically up-regulated when Arabidopsis seeds are imbibed, and thus rapidly depletes the ABA pool and releases the seeds from dormancy. Seeds of the mutant cyp707a2 exhibited hyperdormancy and accumulated six-fold greater ABA content than wild type. Expression of all four CYP707A genes was increased when stressed leaves were rehydrated. Thus, it is clear that expression of the CYP707A genes play a key role in regulating ABA levels. Furthermore, recombinant CYP707A2 protein exclusively oxidized ABA to $8^{\prime}$-hydroxy-ABA and PA. More data shows that other CYP707A genes also have 8' -hydroxylase activity [16-18]. Since both of CYP709B and CYP707A subfamilies are non-A-type cytochrome P450s, and cyp709b3 mutant shows ABA and salt sensitive phenotype, we speculated that the CYP709B subfamily may be involved in ABA catabolism. To address this question, we measured ABA content in seeds and seedlings of wild type and cyp709b3 mutant. As shown in Figure 7 , the ABA content was similar between wild type and cyp $709 b 3$ mutant during seed imbibition and salt treatment. These results rule out a role for the CYP709B3 protein in ABA catabolism during seed germination or salt stress responses.

In order to elucidate the molecular mechanism governing the salt intolerance phenotype in cyp709b3, we analyzed the expression of $\mathrm{ABA}$ and stress-induced marker genes under $150 \mathrm{mM} \mathrm{NaCl}$ treatment. The transcript levels of representative stress-regulated genes (KIN2, RD29A, RD29B, DREB1A and ERD10) in the cyp709b3 mutant were not significantly altered relative to the wild type under salt stress (Figure 6B, C, D, E and F). These data suggest that the cyp $709 b 3$ mutant did not impair the up-regulation of these genes by salt stress. Since ABA content and the expression of stress upregulated marker genes was not affected in the cyp709b3 mutant compared to WT under salt treatment, we therefore conclude that CYP709B3 is not the primary gene involved in ABA signaling or biosynthesis under salt stress. ABA content and the expression of stress up-regulated marker genes reached their highest level at an early time point (before 6 hours); however, the CYP709B3 gene did not increase expression until 24 hours. We therefore suggest that CYP709B3 plays a role in the later stage of salt tolerance. We also searched the expression pattern of CYP709B3 in ABA signaling (abi4-102 and abi1-1) and synthesis deficient mutants (aba1-1) from published microarray data (http://www. genevestigator.com). CYP709B3 expression is not significantly different between ABA mutants and wild type (Additional file 5). According to these results, we conclude that there is not a close relationship between CYP709B3 and ABA signaling or synthesis. Therefore, CYP709B3 may regulate the salt stress response through a novel pathway independent of the wellcharacterized regulators.

\section{Is CYP709B3 involved in salt stress response through alteration of metabolic process?}

When plants are challenged with hyperosmolarity, the osmotic potential in the cell will be increased. To solve this problem, plant cells will accumulate compatible osmolytes to decrease the osmotic potential of the cell cytosol and preserve the activity of enzymes in saline solutions [26]. Various compatible osmolytes, such as proline, polyamines, organic acids and glycine betaine, can greatly reduce stress damage to plant cells [27-32]. The synthesis of compatible osmolytes is often achieved by diversion of basic intermediary metabolites into these unique biochemical reactions, a diversion that is often triggered by stress. In general, cytochrome P450s are involved in primary and secondary metabolism and may be involved in the biosynthesis of some osmolytes. Recently, metabolite profiling analysis was preformed to study metabolic responses to stress in plants $[33,34]$. Other potential biochemical compounds involved in salt tolerance can be identified by metabolic analysis [35-37]. In this report, metabolite profiling analysis was performed to compare the differences in metabolism between wild type and cyp $709 b 3$ under normal and salt stress conditions, and to further identify the substrates of CYP709B3. The initial focus was on compounds whose synthesis or degradation might involve P450 enzymes, which are typically involved in hydroxylation and other oxidative reactions. The metabolomes of both wild type and cyp $709 b 3$ were strongly affected by the salt treatment (Additional files 3 and 4); however, the differences between the lines subjected to similar treatments were relatively subtle. For example, the osmolyte proline had a strong salt-stress response that was similar in direction and magnitude between wild type and cyp709b3 (Additional file 6). Some compounds related to membrane degradation and nucleic acid and protein degradation were increased in cyp $709 \mathrm{b3}$ under salt stress compared to wild type (Figure 8), further confirming the salt intolerance response of the cyp $709 \mathrm{~b} 3 \mathrm{mu}-$ tant. So far, we did not find changes in compounds that would be expected to be synthesized through P450 enzyme activity (hydroxylation and oxidation). We speculate that CYP709B3 possesses a specific, unidentified enzyme activity 
that produces a biochemical compound that regulates salt stress response. Due to the limited number of compounds tested in this report (163 known compounds), further analysis of unknown compounds will provide the information needed to identify the substrate (or substrates) of CYP709B3 and its function in salt tolerance.

\section{Conclusions}

The cyp709b3 null mutant shows an ABA sensitive and salt intolerance phenotype. Expression of the wild type CYP709B3 gene in the cyp709b3 mutant fully complemented the salt intolerance phenotype. The expression of CYP709B3 gene is induced by salt stress. These data demonstrate that the CYP709B3 gene plays a role in the regulation of salt tolerance in Arabidopsis. Further analysis indicates that CYP709B3 may regulate the salt stress response through an unknown pathway independent of the well-characterized regulator.

\section{Methods}

Plant materials, mutants screening and statistical analysis Mutant and wild-type plants in Arabidopsis thaliana ecotype Columbia (Col-0) were used in all experiments. Plants were grown under long day conditions (16 h light/ $8 \mathrm{~h}$ dark) with about $125 \mu \mathrm{E} \mathrm{m} \mathrm{m}^{-2} \mathrm{~s}^{-1}$ light at $22^{\circ} \mathrm{C}$. T-DNA insertion mutants of CYP709B1 (At2g46960), CYP709B2 (At2g46950) and CYP709B3 (At4g27710) were obtained from the Arabidopsis Biological Resource Center [38,39]. Homozygous null mutants were screened by genomic PCR using gene-specific primers. The primer pairs used for identification of mutants are $5^{\prime}$ - gtcaggtgcgttgaaaacttg$3^{\prime}$ and $5^{\prime}$ - tgagatgcatatccttggctc-3' for cyp709b1, 5' - ac tcgttagagcttgcagctg- $3^{\prime}$ and $5^{\prime}$ - ctcctgagcacgatcaatctc- $3^{\prime}$ for cyp709b2-1, 5' - ttgtgagacgatcacgtgaac-3' and 5' - gtcgctatgatatcagcggtc-3' for cyp709b2-2, and 5' - catgagctagcgaaacaggtc-3' and 5' - tttaatcacgggtccgtacag-3' for cyp709b3.

For observation of seedling phenotypes, sterilized seeds were plated on a half-strength Murashige and Skoog (MS) medium with $1 \%$ sucrose and $0.6 \%$ agar. Plates were incubated in a growth chamber at $22^{\circ} \mathrm{C}$ under continuous light $\left(100 \mu \mathrm{E} \mathrm{m}^{-2} \mathrm{sec}^{-1}\right)$. Seedlings were grown in different treatment conditions for phenotypic investigation.

At least two biological repetitions were performed for all experiments. For single experiments, at least three independent repetitions were done. Results from one of the biological repeats that gave comparable results were shown. Student's t test was used to determine whether the difference between two groups of data at a specific time point is statistically significant $(\mathrm{P}<0.05)$. Statistically different data groups are indicated in the relevant figures.

\section{Germination and stress tolerance assays}

For germination assays, around 100 seeds each from wild type and mutants were sown in triplicate on filter paper soaked with distilled water, or with different concentrations of $\mathrm{ABA}$, or on MS plates with the addition of $0,100,150$ or $200 \mathrm{mM} \mathrm{NaCl}$. Seed germination (emergence of radicals) was scored daily. For the salt tolerance assay, around 80 wild type and mutant seeds were germinated on MS agar plates. After 4-days growth, seedlings were transferred onto MS agar plates with addition of different concentrations of $\mathrm{NaCl}$. The seedlings with fully yellow and bleached cotyledon were scored as dead seedlings. Only seedlings that developed true leaves and remained green were scored as survival seedlings. Seedlings were collected at indicated times for real time PCR and phytohormone analysis. Each treatment was performed in triplicate [40].

\section{RNA extraction and real time PCR}

Total RNA was extracted using TRIzol reagent (Invitrogen). After DNase treatment (Turbo DNA-free, Ambion), $1 \mu \mathrm{g}$ of total RNA was used for reverse transcription. Quantitative real time PCR assays were performed using the generated cDNA as template and gene-specific primers. Semi-quantitative RT-PCR was performed to detect transcript levels in wild type and mutants. The primer pairs were $5^{\prime}$ - atgttgtcgaacaagttaggtttc $-3^{\prime}$ and $5^{\prime}$ - gttatccacaggggtgtgct $-3^{\prime}$ for CYP709B1, 5' - cgaccctcacactacacacg-3' and $5^{\prime}$ - cggaaccgttgaagaatcat- $3^{\prime}$ for CYP709B2, $5^{\prime}$ - atggaacttataagcacaatcaatctc- $3^{\prime}$ and $5^{\prime}$ - atactcgggggagaggctaa-3' for CYP709B3, and 5' - cactgtgccaatctacgagg gt $-3^{\prime}$ and $5^{\prime}$ - cacaaacgagggctggaacaag $-3^{\prime}$ for ACTIN2.

Real time PCR was conducted using SYBR Green Fast Mix Rox (Quanta) on a StepOnePlus Real-Time PCR System (Applied Biosystems). Estimates of transcript amount were performed using the comparative threshold cycle method. Relative expression levels were normalized using ACTIN2 as an internal control. The primer pairs (forward and reverse) used for real time PCR were ACTIN2 (At3g18780, 5' - ggtaacattgtgctcagtggtgg-3' and 5' - aacg accttaatcttcatgctgc-3'), CYP709B1 (At2g46960, 5' - ggcatcc atttgtgttgaccagaag- $3^{\prime}$ and $5^{\prime}$ - cttctttatctcgctgaggtttccg- $\left.3^{\prime}\right)$, CYP709B2 (At2g46950, 5' - atgcacagagacaaagccgtttgg-3' and $5^{\prime}$ - ccaatgcaagctcttggtcccatt-3'), CYP709B3 (At4g 27710, 5' - tgttgatggagtcgcttcgtctgt- $3^{\prime}$ and $5^{\prime}$ - tggccttg tctctgtgcatcttca-3'), RD29A (At5g52310, 5' - gttactgatc ccaccaaagaaga- $3^{\prime}$ and $5^{\prime}$ - ggagactcatcagtcacttcca-3'), $R D$ $29 B$ (At5g52300, 5' -acaatcacttggcaccaccgtt-3' and 5' - aact acttccaccggaatccgaa-3') , KIN2 (At5g15970, 5' - gcaac aggcgggaaagagtat- $3^{\prime}$ and $5^{\prime}$ - ccggtcttgtccttcacgaa- $\left.3^{\prime}\right), D R$ EB1A (At4g25480, 5' -gatcagcctgtctcaatttc- $3^{\prime}$ and $5^{\prime}$-cttctg ccatattagccaac-3')and ERD10 (At1g20450, 5' -tctctgaaccagagtcgttt-3' and $5^{\prime}$-cttcttctcaccgtcttcac- $3^{\prime}$ ) 


\section{Quantitative measurement of ABA}

A method used for detection and quantification of acidic plant hormones was developed and performed by the Proteomics \& Mass Spectrometry Facility at the Donald Danforth Plant Science Center. The method was modified according to published reference [41].

\section{Plasmid constructs and plant transformation}

To make the CYP709B3 complementation construct, the full-length CYP709B3 genomic DNA, including the 1547-bp region upstream of the ATG start codon, was PCR-amplified using CYP709B3-Pro-F (5'-ccaaagaaagcaaagccaag- $3^{\prime}$ ) and CYP709B3-R (5'-tccgagagggtgaagcattacg-3') primers. The fragment was cloned into the pCR8/GW/TOPO (Invitrogen) vector. The LR recombination reaction was performed to transfer the fragment to the plant expression vector pMDC99 [42] in order to generate the final construct Pro $_{\text {СYP709B3: }}$ CYP709B3. For the GUS fusion construct, the 1547-bp region upstream of the ATG start codon was amplified by PCR using CYP709B3-Pro-F and CYP709B3-Pro-R (5' - taaaagaaggaacacaagtagctc- $\left.3^{\prime}\right)$ primers and introduced into pCR8/GW/TOPO. Finally, a LR recombination reaction was performed to transfer the fragment to the plant expression vector pMDC162 in order to generate the final construct of Pro $_{\text {СYP709B3: }}$ GUS. All constructs were sequence confirmed. All constructs were introduced into Agrobacterium tumefaciens strain GV3101 and then transformed into wild-type or cyp $709 b 3$ mutant plants by the floral dipping method [43].

\section{GUS staining assay}

GUS staining was performed according to a published method [44].

\section{Metabolite profiling analysis}

Wild type (W) and cyp709b3 (M) seedlings were collected after 2 days (2D) and 4 days (4D) of growth on normal or $150 \mathrm{mM} \mathrm{NaCl} \mathrm{MS} \mathrm{plates.} \mathrm{Sample} \mathrm{extraction,}$ LC/MS and GC/MS analysis were performed as described [34] by METABOLON, Inc. In brief, $100 \mathrm{mg}$ of each sample were extracted using the automated Microlab STAR system. Recovery standards were added prior to the first step in the extraction process. Sample preparation was conducted using a proprietary series of organic and aqueous extractions to remove the protein fraction while allowing maximum recovery of small molecules. The resulting extract was divided into two fractions; one for analysis by LC/MS and one for analysis by GC/MS. Samples were placed briefly on a TurboVap ${ }^{\circledR}$ (Zymark) to remove the organic solvent. Each sample was then frozen and dried under vacuum. Samples were then prepared for the appropriate their instrument, either LC/ MS or GC/MS

\section{Additional files}

\begin{abstract}
Additional file 1: Amino acid alignment of CYP709B1, CYP709B2
and CYP709B3. The analysis was performed using ClustalW2.

Additional file 2: CYP709B3 gene can rescue ABA sensitive phenotype in seed germination. Seeds were sown on wetted filter paper containing $0 \mu \mathrm{M} A B A(A)$ and $1.5 \mu \mathrm{M}$ ABA (B). After 2 days at $4^{\circ} \mathrm{C}$, the plates were placed under continuous light. Germination (emergence of radicals) was scored at indicated times. Error bars indicate SE $(n=3)$. Statistically different to wild type ( $p$ value $<0.05$ ) is indicated using asterisks.

Additional file 3: Metabolomic difference between wild type and cyp709b3, with and without salt treatment. Four-day-old seedlings were transferred onto $150 \mathrm{mM} \mathrm{NaCl}$ plates. Untreated and treated seedlings were collected at 2 days (2D) and 4 days (4D) after treatment. $100 \mathrm{mg}$ of tissue was extracted and analyzed by LC/MS and GC/MS by Metabolon, Inc. Values are the means \pm SD of four replicates and presented as ratios. NS: non-salt treatment; SALT: salt treatment. Heat map of statistically significant biochemicals profiled in this study. Shaded cells indicate $p \leq 0.05$ (red indicates that the mean values are significantly higher for that comparison; green values significantly lower).
\end{abstract}

Additional file 4: Comparison of metabolites between wild type (WT) and cyp709b3 (MUT) under non-salt (N) and salt (S) conditions at day 2 (2D) and day 4 (4D).

Additional file 5: CYP709B3 gene expression in $A B A$ signaling and ABA biosynthesis deficient mutants. From http://www.genevestigator. com.

Additional file 6: Proline analysis in seedling samples. Four-day-old seedlings were transferred onto $150 \mathrm{mM} \mathrm{NaCl}$ plates. Untreated and treated seedlings were collected at 2 days (2D) and 4 days (4D) after treatment. $100 \mathrm{mg}$ of tissue was extracted and analyzed by LC/MS and GC/MS by Metabolon, Inc. Values are the means \pm SD of four replicates. $\mathrm{N}$ : non-salt treatment; S: salt treatment. Y-axis: peak intensity.

\section{Abbreviations}

PCR: Polymerase chain reaction; LC/MS: Liquid chromatography-mass spectrometry; GC/MS: Gas chromatography-mass spectrometry.

\section{Competing interests}

The authors have declared no conflict of interests.

\section{Authors' contributions}

GM and OY conceived the study, designed the experiments and drafted the manuscript. GM, TS and DS performed the experiments. All authors read and approved the final manuscript.

\section{Acknowledgements}

This research is mainly supported by grant from NSF (MCB-0923779), but also by grants from DOE (DE-SC0001295) and USDA (2010-65116-20514) to O. Y.

\section{Author details}

${ }^{1}$ Donald Danforth Plant Science Center, 975 North Warson Road, St. Louis, MO 63132, USA. ${ }^{2}$ Present address: Conagen Inc., 1005 North Warson Road, St., Louis, MO 63132, USA. ${ }^{3}$ Present address: The Pennsylvania State University, 115 Agricultural Sciences and Industries Building, University Park, PA 16802, USA.

Received: 9 December 2012 Accepted: 28 August 2013

Published: 28 October 2013

\section{References}

1. Werck-Reichhart D, Feyereisen R: Cytochromes P450: a success story. Genome Biol 2000, 1(6):REVIEWS3003.

2. Werck-Reichhart D, Bak S, Paquette S: Cytochromes p450. Arabidopsis Book 2002, 1:e0028.

3. Bak S, Beisson F, Bishop G, Hamberger B, Hofer R, Paquette S, WerckReichhart D: Cytochromes p450. Arabidopsis Book 2012, 9:e0144. 
4. Durst F, Nelson DR: Diversity and evolution of plant P450 and P450reductases. Drug Metabol Drug Interact 1995, 12(3-4):189-206.

5. Paquette SM, Bak S, Feyereisen R: Intron-exon organization and phylogeny in a large superfamily, the paralogous cytochrome P450 genes of Arabidopsis thaliana. DNA Cell Biol 2000, 19(5):307-317.

6. Ralston L, Yu O: Metabolons involving plant cytochrome P450s. Phytochem Rev 2006, 5:459-472.

7. Schuler MA, Duan H, Bilgin M, Ali S: Arabidopsis cytochrome P450s through the looking plass a window on plant biochemistry. Phytochem Rev 2006, 5:205-237.

8. Schuler MA, Werck-Reichhart D: Functional genomics of P450s. Annu Rev Plant Biol 2003, 54:629-667.

9. Narusaka Y, Narusaka M, Seki M, Umezawa T, Ishida J, Nakajima M, Enju A, Shinozaki K: Crosstalk in the responses to abiotic and biotic stresses in Arabidopsis: analysis of gene expression in cytochrome P450 gene superfamily by cDNA microarray. Plant Mol Biol 2004, 55(3):327-342.

10. Ehlting J, Provart NJ, Werck-Reichhart D: Functional annotation of the Arabidopsis P450 superfamily based on large-scale co-expression analysis. Biochem Soc Trans 2006, 34(Pt 6):1192-1198.

11. Ehlting J, Sauveplane V, Olry A, Ginglinger JF, Provart NJ, Werck-Reichhart D: An extensive (co-)expression analysis tool for the cytochrome P450 superfamily in Arabidopsis thaliana. BMC Plant Biol 2008, 8:47.

12. Matsuno M, Compagnon V, Schoch GA, Schmitt M, Debayle D, Bassard JE, Pollet B, Hehn A, Heintz D, Ullmann P, et al: Evolution of a novel phenolic pathway for pollen development. Science 2009, 325(5948):1688-1692.

13. Godiard L, Sauviac L, Dalbin N, Liaubet L, Callard D, Czernic P, Marco Y: CYP76C2, an Arabidopsis thaliana cytochrome $\mathrm{P} 450$ gene expressed during hypersensitive and developmental cell death. FEBS Lett 1998, 438(3):245-249.

14. Takei K, Yamaya T, Sakakibara H: Arabidopsis CYP735A1 and CYP735A2 encode cytokinin hydroxylases that catalyze the biosynthesis of trans-Zeatin. J Biol Chem 2004, 279(40):41866-41872.

15. Kushiro T, Okamoto M, Nakabayashi K, Yamagishi K, Kitamura S, Asami T, Hirai N, Koshiba T, Kamiya Y, Nambara E: The Arabidopsis cytochrome P450 CYP707A encodes ABA 8'-hydroxylases: key enzymes in ABA catabolism. EMBO J 2004, 23(7):1647-1656.

16. Millar AA, Jacobsen JV, Ross JJ, Helliwell CA, Poole AT, Scofield G, Reid JB, Gubler F: Seed dormancy and ABA metabolism in Arabidopsis and barley: the role of ABA 8'-hydroxylase. Plant J 2006, 45(6):942-954.

17. Missihoun TD, Schmitz J, Klug R, Kirch HH, Bartels D: Betaine aldehyde dehydrogenase genes from Arabidopsis with different sub-cellular localization affect stress responses. Planta 2011, 233(2):369-382.

18. Umezawa T, Okamoto M, Kushiro T, Nambara E, Oono Y, Seki M, Kobayashi M, Koshiba T, Kamiya Y, Shinozaki K: CYP707A3, a major ABA 8'-hydroxylase involved in dehydration and rehydration response in Arabidopsis thaliana. Plant J 2006, 46(2):171-182.

19. Goda H, Sawa S, Asami T, Fujioka S, Shimada Y, Yoshida S: Comprehensive comparison of auxin-regulated and brassinosteroid-regulated genes in Arabidopsis. Plant Physiol 2004, 134(4):1555-1573.

20. Pan Y, Michael TP, Hudson ME, Kay SA, Chory J, Schuler MA: Cytochrome P450 monooxygenases as reporters for circadian-regulated pathways. Plant Physiol 2009, 150(2):858-878.

21. Kandel S, Morant M, Benveniste I, Blee E, Werck-Reichhart D, Pinot F: Cloning, functional expression, and characterization of CYP709C1, the first sub-terminal hydroxylase of long chain fatty acid in plants. Induction by chemicals and methyl jasmonate. J Biol Chem 2005, 280(43):35881-35889.

22. Kandel S, Sauveplane V, Compagnon V, Franke R, Millet Y, Schreiber L, Werck-Reichhart D, Pinot F: Characterization of a methyl jasmonate and wounding-responsive cytochrome P450 of Arabidopsis thaliana catalyzing dicarboxylic fatty acid formation in vitro. FEBS J 2007, 274(19):5116-5127.

23. Winter D, Vinegar B, Nahal H, Ammar R, Wilson GV, Provart NJ: An "Electronic Fluorescent Pictograph" browser for exploring and analyzing large-scale biological data sets. PLoS One 2007, 2(8):e718.

24. Xiong L, Zhu JK: Regulation of abscisic acid biosynthesis. Plant Physiol 2003, 133(1):29-36.

25. Duan H, Huang MY, Palacio K, Schuler MA: Variations in CYP74B2 (hydroperoxide lyase) gene expression differentially affect hexenal signaling in the Columbia and Landsberg erecta ecotypes of Arabidopsis. Plant Physiol 2005, 139(3):1529-1544.
26. Xiong L, Zhu JK: Salt tolerance. Arabidopsis Book 2002, 1:e0048.

27. Burg MB, Ferraris JD: Intracellular organic osmolytes: function and regulation. J Biol Chem 2008, 283(12):7309-7313.

28. Kol S, Merlo ME, Scheltema RA, de Vries M, Vonk RJ, Kikkert NA, Dijkhuizen L, Breitling R, Takano E: Metabolomic characterization of the salt stress response in Streptomyces coelicolor. App/ Environ Microbiol 2010, 76(8):2574-2581

29. Liu J, Zhu JK: Proline accumulation and salt-stress-induced gene expression in a salt-hypersensitive mutant of Arabidopsis. Plant Physiol 1997, 114(2):591-596

30. Strizhov N, Abraham E, Okresz L, Blickling S, Zilberstein A, Schell J, Koncz C, Szabados L: Differential expression of two P5CS genes controlling proline accumulation during salt-stress requires $A B A$ and is regulated by $A B A 1$, ABI1 and AXR2 in Arabidopsis. Plant J 1997, 12(3):557-569.

31. Verslues PE, Sharma S: Proline metabolism and its implications for plant-environment interaction. Arabidopsis Book 2010, 8:e0140.

32. Yamaguchi K, Takahashi Y, Berberich T, Imai A, Miyazaki A, Takahashi T, Michael A, Kusano T: The polyamine spermine protects against high salt stress in Arabidopsis thaliana. FEBS Lett 2006, 580(30):6783-6788.

33. Bednarek P, Pislewska-Bednarek M, Svatos A, Schneider B, Doubsky J, Mansurova M, Humphry M, Consonni C, Panstruga R, Sanchez-Vallet A, et al: A glucosinolate metabolism pathway in living plant cells mediates broad-spectrum antifungal defense. Science 2009, 323(5910):101-106

34. Oliver MJ, Guo L, Alexander DC, Ryals JA, Wone BW, Cushman JC: A sister group contrast using untargeted global metabolomic analysis delineates the biochemical regulation underlying desiccation tolerance in Sporobolus stapfianus. Plant Cell 2011, 23(4):1231-1248.

35. Ashraf M, Harris PJC: Potential biochemical indicators of salinity tolerance in plants. Plant Sci 2004, 166:3-16.

36. Wu W, Zhang Q, Zhu Y, Lam HM, Cai Z, Guo D: Compartive metabolic profiling reveals secondary metabolites correlated with soybean salt tolerance. J Agric Food Chem 2008, 56:11132-11138.

37. Yeo A: Molecular biology of salt tolerance in the context of whole-plant physiology. J Exp Bot 1998, 49(323):915-929.

38. Alonso JM, Stepanova AN, Leisse TJ, Kim CJ, Chen H, Shinn P, Stevenson DK, Zimmerman J, Barajas P, Cheuk R, et al: Genome-wide insertional mutagenesis of Arabidopsis thaliana. Science 2003, 301(5633):653-657.

39. Sessions A, Burke E, Presting G, Aux G, McElver J, Patton D, Dietrich B, Ho P, Bacwaden J, Ko C, et al: A high-throughput Arabidopsis reverse genetics system. Plant Cell 2002, 14(12):2985-2994.

40. Xiong L, Gong Z, Rock CD, Subramanian S, Guo Y, Xu W, Galbraith D, Zhu JK: Modulation of abscisic acid signal transduction and biosynthesis by an Sm-like protein in Arabidopsis. Dev Cell 2001, 1(6):771-781.

41. Chen Q, Zhang B, Hicks LM, Wang S, Jez JM: A liquid chromatographytandem mass spectrometry-based assay for indole-3-acetic acid-amido synthetase. Anal Biochem 2009, 390(2):149-154.

42. Curtis MD, Grossniklaus U: A gateway cloning vector set for highthroughput functional analysis of genes in planta. Plant Physiol 2003 133(2):462-469.

43. Clough SJ, Bent AF: Floral dip: a simplified method for Agrobacteriummediated transformation of Arabidopsis thaliana. Plant J 1998, 16(6):735-743

44. Mao G, Wang R, Guan Y, Liu Y, Zhang S: Sulfurtransferases 1 and 2 play essential roles in embryo and seed development in Arabidopsis thaliana. J Biol Chem 2011, 286(9):7548-7557.

doi:10.1186/1471-2229-13-169

Cite this article as: Mao et al:: CYP709B3, a cytochrome P450 monooxygenase gene involved in salt tolerance in Arabidopsis thaliana. BMC Plant Biology 2013 13:169. 